\title{
Controlled islanding strategy considering power system restoration constraints
}

DOI:

10.1109/PESGM.2012.6344599

Link to publication record in Manchester Research Explorer

\section{Citation for published version (APA):}

Quiros Tortos, J., Tortos, J. Q., \& Terzija, V. (2012). Controlled islanding strategy considering power system restoration constraints. In IEEE Power and Energy Society General Meeting/IEEE Power Energy Soc. Gen. Meet. IEEE. https://doi.org/10.1109/PESGM.2012.6344599

\section{Published in:}

IEEE Power and Energy Society General Meeting|IEEE Power Energy Soc. Gen. Meet.

\section{Citing this paper}

Please note that where the full-text provided on Manchester Research Explorer is the Author Accepted Manuscript or Proof version this may differ from the final Published version. If citing, it is advised that you check and use the publisher's definitive version.

\section{General rights}

Copyright and moral rights for the publications made accessible in the Research Explorer are retained by the authors and/or other copyright owners and it is a condition of accessing publications that users recognise and abide by the legal requirements associated with these rights.

\section{Takedown policy}

If you believe that this document breaches copyright please refer to the University of Manchester's Takedown Procedures [http://man.ac.uk/04Y6Bo] or contact uml.scholarlycommunications@manchester.ac.uk providing relevant details, so we can investigate your claim.

\section{OPEN ACCESS}




\title{
Controlled Islanding Strategy Considering Power System Restoration Constraints
}

\author{
J. Quirós Tortós, Student Member, IEEE, V. Terzija, Senior Member, IEEE
}

\begin{abstract}
This paper proposes a methodology to split the power system across the weak areas of the network affected by a large disturbance, which might lead to a total system blackout. The final splitting strategy is carried out by opening the transmission lines with minimum power exchanged, i.e. by minimising the power exchange between areas. Since one or more of the created islands might reach an unstable operating point, and therefore, cause a power system blackout, the proposed methodology includes at least one blackstart unit within each island and assures sufficient generation capability to match the load consumption within each island. By assuring blackstart availability and sufficient generation capability, parallel power system restoration is planned in case of any eventuality. For validation purposes, the methodology is implemented and tested on the IEEE 9-bus and 118-bus test systems.
\end{abstract}

Index Terms--Controlled islanding, parallel power system restoration, slow coherency, weak areas, weak connection, wide area monitoring, protection, and control (WAMPAC).

\section{INTRODUCTION}

$\mathrm{I}^{\mathrm{N}}$ NTENTIONAL controlled islanding is an efficient tool for avoiding system wide instabilities, cascading outages and blackouts. When the power system integrity cannot be maintained, splitting strategies are carried out to separate the system into smaller subsystems, also known as islands. This is commonly performed in order to create stable islands with the minimum possible load-generation mismatch, maintaining generator coherences and other static and dynamic constraints [1], [2].

Different methods have been proposed to determine possible splitting strategies i.e. to determine where to split the power system. These methods have mainly taken into consideration the generator coherence and the load-generation balance. Nonetheless, the introduction of Parallel Power System Restoration (PPSR) constraints in controlled islanding strategies is still an unexplored research area and a practical engineering challenge.

Although the objective of controlled islanding schemes is to avoid a complete blackout, one or more islands might reach this unstable operating point after the splitting strategy is

This work was supported in part by the Engineering and Physical Science Research Council (EPSRC) in the UK and The University of Costa Rica.

J. Quirós Tortós and V. Terzija are with the School of Electrical \& Electronic Engineering, University of Manchester, Manchester, UK (e-mail: jairoquirostortos@ieee.org, terzija@ieee.org). carried out. When this undesirable event occurs in an island i.e. when a local blackout occurs, PPSR should be carried out in order to restore the island, and therefore, restore the power system. It has been stated in [3] that the following constraints, regarding the PPSR, should be considered when applying controlled islanding strategies.

$>$ Each island should have sufficient blackstart (BS) capability,

$>$ Each island should have enough cranking paths to crank non-blackstart units or pick-up loads,

$>$ Each island should have the ability to match generation and load within prescribed frequency limits,

$>$ Each island should have adequate voltage controls to maintain a suitable voltage profile,

$>$ All tie points for subsystems must be capable of synchronization with adjacent subsystems,

$>$ All islands should share information with other islands.

Several methodologies to split the power system into smaller islands are based on the slow coherency theory. For example, in [4], a slow coherency method based on two-times scale theory is used to create islands after large disturbances. The paper states that small changes in the splitting strategy are obtained across an area when the location, the size of the disturbance, and loading conditions change.

In [5], a slow coherency technique is used to group both generators and load buses into a single group considering the closeness of each other. This approach uses the slow eigenbasis theory to collect coherent groups. This methodology determines the closeness of each load bus $j$ to the reference generator $i$, by applying the cosine function of the angle between the two row vectors of the eigenbasis matrix corresponding to buses $i$ and $j$. In [6], a method based on the weak coupling concept for identifying groups of slowly coherent generators is presented.

Most recently, a real time approach for finding proper system splitting strategies using a three-phase Ordered Binary Decision Diagram (OBDD) method is proposed in [7]. A similar approach is followed in [8], where a two-phase OBDD algorithm is used to identify the splitting points to separate the power system. In the first phase, synchronism of machines and power balance within areas are assured by the methodology. Then, using power flow analysis, the rated values and limits are verified for the possible solution obtained in the first step. Since these approaches take into consideration only steadystate constraints, an approach considering transient simulations is presented in [9]. 
The main drawbacks of the aforementioned solutions are the long computational time required to obtain the splitting strategy and the lack of PPSR planning stage. In order to solve these disadvantages, this paper identifies the weak areas in the power system, subject to have at least one BS unit, and to be able to match the load consumption, in each island. By computing the eigenbasis matrix, the weak areas are defined as the areas from the weak connection points up to a predefined threshold. The weak areas are comprised of the transmission lines where the network is most likely to be split when a large disturbance occurs.

By computing the power exchanged across these weak links, the splitting strategy is carried out by opening the transmission lines with minimum power exchanged between areas. In real case system, the power exchanged between areas can be obtained by using modern communication and sensor technology, e.g. Global Positioning System (GPS) synchronized Phasor Measurement Units (PMUs), installed across the power system, which result in Wide Area Monitoring Protection and Control (WAMPAC) systems [10]. Therefore, the proposed methodology might be a new WAMPAC application.

By applying the proposed methodology, strong connected islands are created, transient oscillations between areas are reduced, and better load-generation balance is obtained. Moreover, blackstart availability is assured and the load consumption can be met by the available units within each island. Results for the IEEE 9-bus and 118-bus test systems are presented to validate and demonstrate the proposed methodology.

Section II presents the concept of controlled islanding combined with a new approach of PPSR planning. Power system modelling and constraints are also included in this section. The splitting methodology based on the minimisation of the power exchanged between areas across the weak links is presented in Section III. The simulations results for the IEEE 9-bus and 118-bus test systems are presented in Section IV. Finally, the conclusions drawn from the study are provided in Section V.

\section{Controlled Islanding AND Parallel Power System RESTORATION}

Methodologies to split the power system into smaller islands are carried out when groups of generators have lost synchronism. Identifying stable islands with minimum possible load-generation mismatch and considering generators coherences have been the main objectives during the intentional controlled islanding.

Nevertheless, methodologies to split the power system considering PPSR constraints are still an unexplored field and a practical engineering challenge. As the created islands may be unstable, and therefore, cause a local blackout, the PPSR methodology should always be taken into consideration when applying controlled islanding strategies.

This paper represents a power system with $N_{b}$ buses, $N_{g}$ generators and $R$ BS units as an undirected finite graph
$\mathbf{G}\left(\mathbf{V}, \mathbf{E}, \mathbf{V}_{\mathbf{G}}, \mathbf{B}, \mathbf{W}\right)$ where $\mathbf{V}=\left\{v_{1}, v_{2}, \ldots, v_{N_{b}}\right\}$ denotes the set nodes (buses in a power system) and $\mathbf{E}$, with elements $e_{i j}\left(i, j=1,2, \ldots, N_{b}\right)$, represents the set of edges (transmission lines in a power system). The generators directly connected are contained in a subset $\mathbf{V}_{\mathbf{G}} \subset \mathbf{V}$, while the BS units are contained in $\mathbf{B}$. $\mathbf{W}$ is a set of weight factors associated with each edge (it is represented as the average power flow in each transmission line).

\section{A. Dynamic modelling}

Since excitation and governor controllers only modified the damping of the transients but do not change natural frequencies, the information about the coherency can be obtained using a linearised second-order system to model a $N_{g}$-machine power system in the slow coherency theory as follows [11]:

$$
\begin{gathered}
\ddot{\mathbf{x}}=\mathbf{A} \mathbf{x}=\left(\mathbf{M}^{-1} \mathbf{K}\right) \mathbf{x} \\
\mathbf{x}=\left[\Delta \delta_{1}, \Delta \delta_{2}, \ldots, \Delta \delta_{N_{g}}\right]^{\mathrm{T}} \\
\mathbf{A}=\mathbf{M}^{-1} \mathbf{K} \\
\mathbf{M}=\operatorname{diag}\left(2 H_{1} / \omega_{0}, 2 H_{2} / \omega_{0}, \ldots, 2 H_{N_{g}} / \omega_{0}\right) \\
K_{i j}=\left\{\begin{array}{c}
-\left|V_{i}\right|\left|V_{j}\right|\left[\begin{array}{c}
B_{i j} \cos \left(\delta_{i}-\delta_{j}\right)- \\
G_{i j} \sin \left(\delta_{i}-\delta_{j}\right)
\end{array}\right], i \neq j \\
-\sum_{l=1, l \neq i}^{n} K_{i l},
\end{array}\right.
\end{gathered}
$$

where $V_{i}$ and $V_{j}$ are the bus voltage magnitude at bus $i$ and $j$ respectively. $\delta_{i}$ is the rotor angle in radians and $H_{i}$ is the inertia constant in seconds of the $i$-th machine; $G_{i j}$ and $B_{i j}$ are the real and imaginary entries of the admittance matrix $\mathbf{Y}_{\text {bus. }} \mathbf{K}$ matrix is also called the connection matrix and it contains the strong internal $\left(\mathbf{K}^{\mathbf{I}}\right)$ and the weak external $\left(\mathbf{K}^{\mathbf{E}}\right)$ connections.

$$
\mathbf{K}=\mathbf{K}^{\mathbf{I}}+\mathbf{K}^{\mathbf{E}}
$$

It is commonly assumed that the state variables $\mathbf{x}$ are divided into two types: internal for connections between the states in the same areas and external for connections between the states in different areas. Thus, a partition of a dynamic network into $r$ areas is the partition of the states of $\mathbf{x}$ into $r$ sets such that every state $\mathbf{x}_{\mathbf{i}}$ is assigned to one and only one set [12].

The bisection case is initially used in this paper to split the network into two sub-graphs, $\mathbf{G}_{\mathbf{1}}\left(\mathbf{V}_{\mathbf{1}}, \mathbf{E}_{\mathbf{1}}, \mathbf{V}_{\mathbf{G} \mathbf{1}}, \mathbf{B}_{\mathbf{1}}, \mathbf{W}_{\mathbf{1}}\right)$ and $\mathbf{G}_{\mathbf{1}}\left(\mathbf{V}_{\mathbf{2}}, \mathbf{E}_{\mathbf{2}}, \mathbf{V}_{\mathbf{G} 2}, \mathbf{B}_{2}, \mathbf{W}_{\mathbf{2}}\right)$. Each sub-graph represents a subsystem of the original power system. The splitting strategy for large power systems will require recursive bisection when it is split into more than two islands. Here, $\mathbf{G}_{\mathbf{1}}$ and $\mathbf{G}_{\mathbf{2}}$ must satisfy:

$$
\begin{aligned}
& \mathbf{G}_{1} \cap \mathbf{G}_{\mathbf{2}}=\varnothing \\
& \mathbf{G}_{\mathbf{1}} \cup \mathbf{G}_{\mathbf{2}}=\mathbf{G}
\end{aligned}
$$

\section{B. Constraints considered for intentional controlled islanding}

Intentional controlled islanding should be strategically planned, with a focus on creating strong connected islands 
with the minimum possible load-generation mismatch. Since reactive power balance can usually be controlled via local compensation, the active power balance is the main issue when ensuring stable subsystems after large disturbances and islanding actions.

The proposed methodology determines the weak areas across the power system. Then, by computing the power exchanged in the transmission lines within these weak regions, the splitting strategy is carried out by opening the transmission lines with minimum power exchanged. By opening these transmission lines, transient oscillations between areas are reduced and better load-generation balance is obtained. Also, as these open transmission lines are within the weak areas of the power system, strong connected islands are created.

Two other constraints are the availability of BS units within each island and sufficient active power from generators to match loads within each island. Since the stability margins are lower than the one for the entire power system, created islands are always exposed to reach the blackout. Hence, it is important to assure at least one of these units within each island and to be able to match load within prescribed frequency limits. This can also be viewed as a PPSR planning stage.

\section{Splitting Strategy Based on Weak Area IDENTIFICATION}

The close relationship existing between slow coherency and weak connection has been discussed in [12]. In fact, the concept of slow coherency is based on the theory that after a large disturbance occurs in a multi-machine power system, groups of generators have a tendency to swing together.

The identification of the weakest connections across the power system is solved by slow coherency theory. These weakest connections are functions of the admittance matrix parameter, machine inertias and the initial rotor angles of the interconnected machines. Therefore, it can be noticed that the boundaries encountered for each island depend upon the inherent structural characteristics of the power system [13]. Moreover, the availability of inter-tie lines, a request for the load-generation balance and existence of at least one BS unit within each island must be considered when creating these islands.

The slow coherency theory assumes that the state variables of an $n$-th order system are divided into $r$ slow states and $(n-r)$ fast states, namely $y$ and $z$, respectively, where the $r$ slowest states represent $r$ groups with the slow coherency [12]. This is based on the singular perturbation form and it can be formulated as follows.

$$
\begin{array}{ll}
\frac{d y}{d t}=f(y, z, t), & y\left(t_{0}\right)=y_{0} \\
\frac{d z}{d t}=G(y, z, t), & \mathrm{z}\left(t_{0}\right)=z_{0}
\end{array}
$$

The assumption that the coherent groups of generators are independent of the size of the disturbance and the consideration that the coherent groups are independent of the level of detail used to model the generating units are commonly considered in order to carry out the slow coherency analysis [4]. Based on this second assumption, the linearised model presented in Section II-A can be used.

The weak connection form is another concern commonly linked to the slow coherency. In fact, the slow coherency phenomenon occurs in dynamic networks when the connections between areas are weak [12]. When the dynamic properties of a two area system can be described by (11), it is said that this system is weakly connected.

$$
\varepsilon\left[\begin{array}{c}
\frac{d \mathbf{x}_{1}}{d t} \\
\frac{d \mathbf{x}_{2}}{d t}
\end{array}\right]=\left[\begin{array}{c}
\frac{d \mathbf{x}_{1}}{d \tau} \\
\frac{d \mathbf{x}_{2}}{d \tau}
\end{array}\right]=\left[\begin{array}{cc}
\mathbf{A}_{11}+\varepsilon \mathbf{A}_{11}^{\prime} & \varepsilon \mathbf{A}_{12} \\
\varepsilon \mathbf{A}_{21} & \mathbf{A}_{22}+\varepsilon \mathbf{A}_{22}^{\prime}
\end{array}\right]\left[\begin{array}{l}
\mathbf{x}_{1} \\
\mathbf{x}_{2}
\end{array}\right]
$$

In (11), $\mathbf{x}_{1}$ and $\mathbf{x}_{2}$ are $n_{1}$ and $n_{2}$ column vectors, $\mathbf{A}_{11}, \mathbf{A}_{11}^{\prime}$, $\mathbf{A}_{12}, \mathbf{A}_{21}, \mathbf{A}_{22}, \mathbf{A}_{22}^{\prime}$ are matrices of order one without any zero entries, $\tau=\left(t-t_{0}\right) / \varepsilon$ and $\varepsilon$ is a small positive parameter in the slow coherency solution when the external connections are weak or sparse.

In [14], a linear analysis is carried out to prove that by selecting the $r$ slowest modes, the aggregated system will have the weakest connections between different groups of generators. The weak connection form best states the reason for islanding based on slow coherency grouping [13].

Groups of strongly interacting units with weak connections between groups or areas are always encountered in real large scale power systems. In fact, when a large disturbance occurs, it is imperative to disconnect these weak connections before the slow interaction becomes significant.

The methodology for system splitting based on weak connections, which is a system property independent of operating conditions or the degree of modelling, minimises the dynamic coupling between islands according to the following formula.

$$
\min (\underbrace{\underbrace{\sum_{v_{i} \in \mathbf{V}_{L}^{l}, v_{j} \in \mathbf{V}_{L}^{k}, l \neq k}\left(\frac{\partial P_{i j}}{\partial \theta_{i j}}\right)}_{\forall_{2}}}_{\underbrace{}_{\Psi_{1}} \sum_{\underbrace{}_{m} \in \mathbf{V}_{G}^{l}, v_{n} \in \mathbf{V}_{G}^{k}, l \neq k} \frac{1}{2}\left(\frac{\partial P_{m n}}{\partial \theta_{m n}}\right)\left(\frac{1}{H_{m}}+\frac{1}{H_{n}}\right)})
$$

subject to,

$$
\begin{gathered}
\exists B \in \mathbf{B} \mid B_{i} \in \mathbf{V}_{\mathbf{G} 1} \vee B_{j} \in \mathbf{V}_{\mathbf{G} 2} \quad i \neq j=1,2, \ldots, B \\
\sum_{i=1}^{N_{g}^{r}} P_{g, i}^{\min , r} \leq P_{L}^{r} \leq \sum_{i=1}^{N_{g}^{r}} P_{g, i}^{\max , r}
\end{gathered}
$$

In (12), $\psi_{1}$ denotes the dynamic coupling between generators, which is normalised by the inertias and $\psi_{2}$ denotes the dynamic coupling between load buses. $V_{G}^{l}$ and $V_{G}^{k}$ represent the group of voltages at generator buses within islands $l$ and $k$, respectively. $V_{L}^{l}$ and $V_{L}^{k}$ represent the group of voltages at load buses within islands $l$ and $k$, respectively. By including (13) in the method, there is at least one BS unit 
within each island and the load will be matched by the available units within each island $r$.

As the weak connections theory identifies only one splitting strategy, the concept of weak areas is introduced in [13]. This approach is based on the eigenbasis matrix of the power system and it provides several splitting options of the power system into smaller subsystems. The final splitting strategy can be obtained across these regions based on another criterion. The weak areas are defined as the areas from the weak connections points up to a pre-defined threshold [13].

When the weak areas are identified, the power exchange in these weak links is computed. The final splitting strategy will be based on the following criteria.

$$
\min _{\mathbf{v}_{1}, \mathbf{v}_{2} \subset \mathbf{v}_{i \in \mathbf{V}_{\mathbf{1}}, j \in \mathbf{V}_{\mathbf{2}}}} \frac{\left|P_{i j}\right|+\left|P_{j i}\right|}{2}
$$

where $P_{i j}$ and $P_{j i}$ are the active power in the transmission line connecting buses $i \in \mathbf{V}_{\mathbf{1}}$ and $j \in \mathbf{V}_{\mathbf{2}}$. By applying (14), transient oscillations are reduced, better load-generation balance is obtained and strong connected islands are created.

\section{A. Lists of assumptions and criteria considered}

Given a power system as described in Section II, some assumptions considered in this paper are:

1) Availability of all BS units. Thus, each island would have at least one BS unit.

2) All machines are able to operate at full rated power. Thus, there would be enough generation capability within each island.

3) Each island has adequate voltage controls to maintain a suitable voltage profile.

4) Sufficient information about the power flow in the weak links can be obtained from WAMPAC systems.

Based on these assumptions, this paper uses the following criteria: the identification of weak areas. Which is done by defining a threshold beyond the weak connections (12). And the other criterion is the minimisation of the power exchange between strong connected islands (14).

It is important at this stage to mention that the proposed splitting strategy does not consider contingency in each island. Instead, it is based on the fact that after a large disturbance, groups of generators have a tendency to swing together. Once these oscillations are obtained and identified, the proposed method will determine the weak area and then the final splitting strategy within these regions.

\section{Simulation Results}

The proposed splitting methodology is demonstrated and validated using the IEEE 9-bus 118-bus test systems. Simulations are performed using MATLAB 7.10 (R2010a) on an Intel ${ }^{\circledR}$ Core $^{\mathrm{TM}} 2$ Duo CPU E7500 @ $2.93 \mathrm{GHz}, 4$ GB of RAM.

\section{A. Test case I: IEEE 9-bus test system}

The proposed method is firstly explained in detail using the IEEE 9-bus test system shown in Fig. 1 [15]. This test system contains 3 synchronous generators, 6 transmission lines, 3 transformers and 3 constant power loads. Generator data are provided in the Appendix. In this case, it is assumed that generators 1 and 2 are BS units. In the first step, the slow coherency analysis [12] is carried out. Here, generators 2 and 3 are determined as coherent generators. Consequently, the other group is only generator 1 .

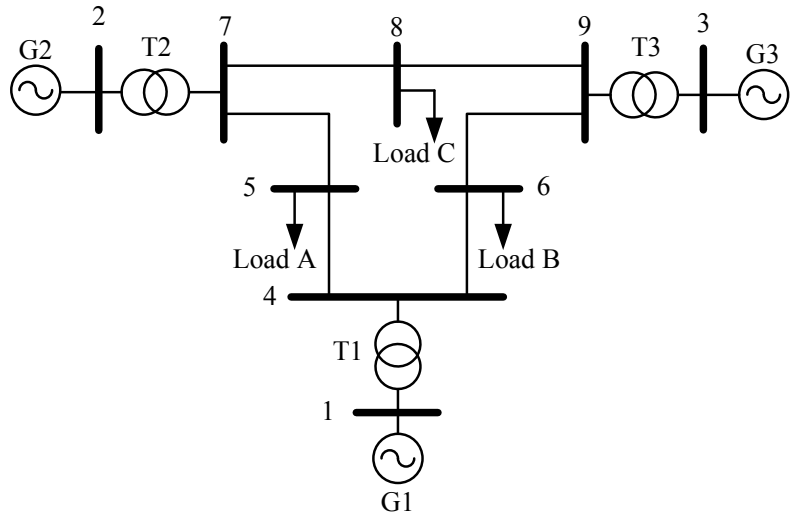

Fig. 1. Single line diagram of the IEEE 9-bus test system

The graph for all $n=9$ nodes is obtained in the second step. Then, by applying the weak connection algorithm (12), it can be determined that the weak connections are branches $4-5$ and 4-6.

The weak connections algorithm (12) is further extended to determine the weak areas as shown in Fig. 2(a). This is achieved by defining a threshold, in the eigenbasis matrix, beyond the weak connections of 20 per cent ( 10 per cent on each side of the weak connection).

By running a conventional power flow, (i.e. using Newton Raphson solution), the power flow in the weak links is computed. The average power flow $\left(\left(\left|P_{i j}\right|+\left|P_{j i}\right|\right) / 2\right)$ in the branches within the weak area is shown in Fig.2(b). This is used as the weight factor associated with the weak links. The weight factor in each edge is used in the objective function (14) to determine the final splitting strategy. As it can be noticed in Fig. 2(b), by splitting the graph across edges 4-5 and 6-9, the cost function is $(40.8+60.1)=100.9$. On the other hand, by splitting the graph across edges $4-5$ and $4-6$, the cost function is $(40.8+30.6)=71.4$.

Therefore, the final splitting strategy, possessing the lowest power exchange is represented as a dashed line in Fig. 2(b). By performing this splitting strategy, two different islands are created: first $\{1,4\}$ and second $\{2,3,5,6,7,8,9\}$. The load and generation in each island are presented in Table I. BS availability and generation capability in each island are also presented in Table I. As it can be noticed, at least one BS unit and sufficient generation capability are available in each island. It is important to understand that as the weak connection algorithm is used, strong connected islands are determined. Finally, the computational time required to determine the final splitting strategy was only $3.5 \mathrm{~ms}(2.2 \mathrm{~ms}$ for the weak area identification and $1.3 \mathrm{~ms}$ for the final splitting strategy and the power flow computation). 


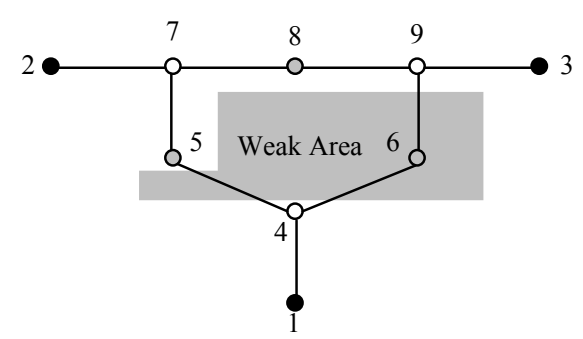

(a)

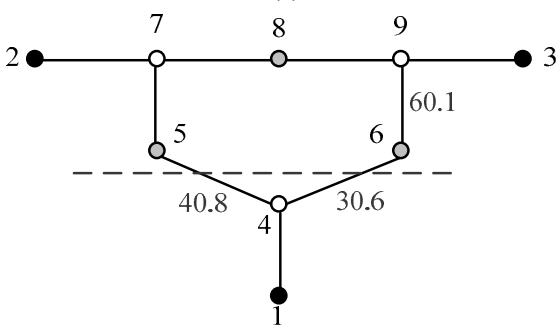

(b)

Fig. 2. Graph representation of the IEEE 9-bus (a) presenting the weak areas (b) presenting the weight factors and the final splitting strategy

TABLE I

LOAD AND GENERATION WITHIN EACH ISLAND FOR THE IEEE 9-BUS TEST SYSTEM

\begin{tabular}{cccccc}
\hline \hline Island & $\begin{array}{c}\Sigma P_{L} \\
(M W)\end{array}$ & $\begin{array}{c}\Sigma P_{G} \\
(M W)\end{array}$ & $\begin{array}{c}\Sigma P_{L}-\Sigma P_{G} \\
(M W)\end{array}$ & $\begin{array}{c}\text { Available } \\
\text { BS unit }\end{array}$ & $\begin{array}{c}\text { Available } \\
\text { active power } \\
(M W)\end{array}$ \\
\hline \hline 1 & 0 & 71.6 & -71.6 & 1 & 250 \\
2 & 315 & 248 & 67 & 2 & 570 \\
\hline \hline
\end{tabular}

\section{B. Test case II: IEEE 118-bus test system}

In order to demonstrate and validate the proposed methodology in a larger system, the IEEE 118-bus shown in Fig. 3 is now used in this paper. This test system contains 19 synchronous generators, 177 transmission lines, 9 transformers and 91 constant power loads [16]. Generator data are provided in the Appendix. This paper considers generators $10,25,69,87$ and 89 as BS units.

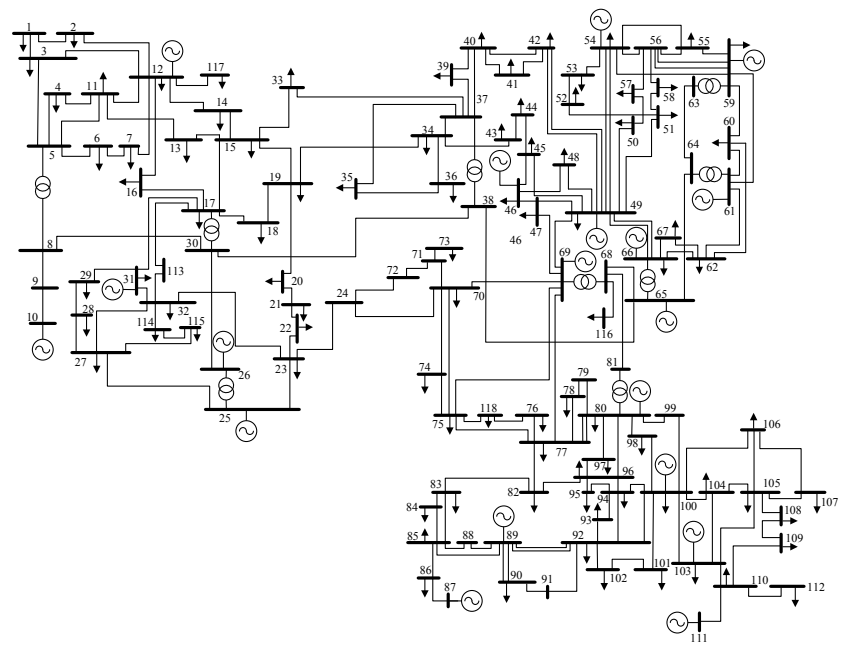

Fig. 3. Single line diagram of the IEEE 118-bus test system

Table II is obtained in the first step by using the slow coherency algorithm [12] and by changing the number of coherent groups of generators from two groups up to the number of available BS units.
TABLE II

COHERENT GENERATORS GROUPS FOR THE IEEE 118-BUS TEST SYSTEM SPLIT INTO DIFFERENT SUBSYSTEMS

\begin{tabular}{|c|c|c|c|c|c|}
\hline$r$ & \multicolumn{5}{|c|}{ Coherent Generator groups } \\
\hline 2 & \multicolumn{2}{|c|}{$10,12,25,26,31$} & \multicolumn{3}{|c|}{$\begin{array}{c}46,49,54,59,61,65,66,69,87,80,89 \\
100,103,111\end{array}$} \\
\hline 3 & \multicolumn{2}{|c|}{$10,12,25,26,31$} & $\begin{array}{c}46,49,54,59,61 \\
65,66,69\end{array}$ & \multicolumn{2}{|c|}{$\begin{array}{c}87,80,89,100,103, \\
111\end{array}$} \\
\hline 4 & \multicolumn{2}{|c|}{$10,12,25,26,31$} & $\begin{array}{c}46,49,54,59,61, \\
65,66,69\end{array}$ & 87 & $\begin{array}{c}80,89,100 \\
103,111\end{array}$ \\
\hline 5 & 10,12 & $25,26,31$ & $\begin{array}{c}46,49,54,59,61 \\
65,66,69\end{array}$ & 87 & $\begin{array}{c}80,89,100 \\
103,111\end{array}$ \\
\hline
\end{tabular}

In the second step, the graph for all $n=118$ nodes is obtained. This graph is shown in Fig 4. Then, by computing the eigenbasis matrix, and by changing the number of islands, the weak connections methodology (12) is carried out. Here, the constraints of having at least one BS unit and being able to match the load with the generation capability in each island are assured by including (13) in the methodology.

By identifying the weak connections and defining a threshold, in the eigenbasis matrix, beyond the weak connections of 25 per cent (12.5 per cent on each side of the weak connections), the weak areas across the power system can be determined as shown in Fig. 5.

When the weak areas are known, the power flow exchange in the weak links between the areas is computed by running a conventional power flow. The power flow method is applied to the base case obtained from [16]. Using the proposed methodology, the space search is considerably reduced, since the power flow in the remaining transmission lines does not have to be included. Then, by applying the objective function (14), the final splitting strategy can be determined for the specific number of areas.

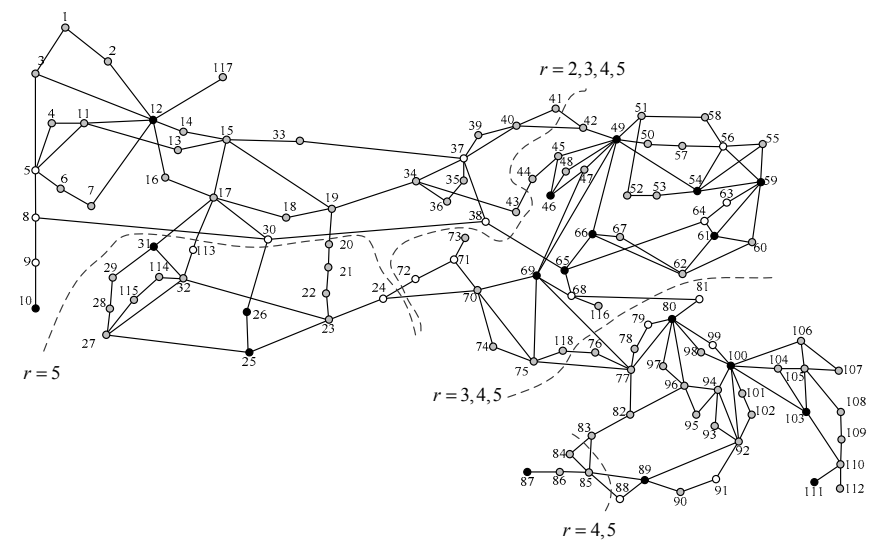

Fig. 4. Graph representation of the IEEE 118-bus test system representing the weak connections

1) Splitting the IEEE 118-bus test system into two areas

Assuming that a large disturbance in the IEEE 118-bus test system produces two different groups of generators (this disturbance might be a three-phase fault near bus 25 at line 23-25), the cut-set to split the network into two islands is determined. Thus, by using (14), the splitting strategy is found as shown in Table III and Fig. 6. Table III also presents the total interrupted power flow when carrying out this splitting strategy i.e. the objective function value. The load and the 
generation in each island are presented as well.

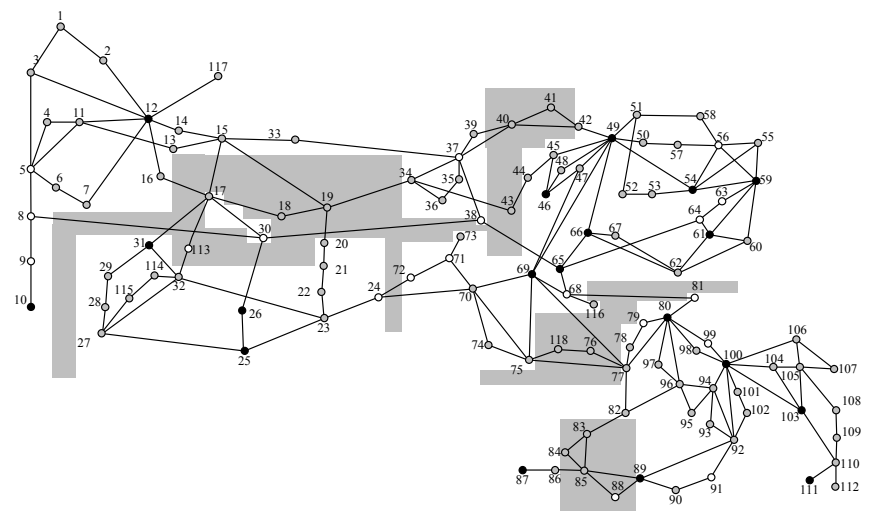

Fig. 5. Weak areas for the IEEE 118-bus test system

TABLE III

SPlitTing Strategy For THE IEEE 118-Bus Test System Split InTO Two ISLANDS

\begin{tabular}{ccccccc}
\hline \hline Cut-set & $\sum \frac{\left|P_{i j}\right|+\left|P_{j i}\right|}{2}$ & \multicolumn{2}{c}{ Area 1 } & \multicolumn{2}{c}{ Area 2 } \\
& $\begin{array}{c}\Sigma P_{L} \\
(M W)\end{array}$ & $\begin{array}{c}\Sigma P_{G} \\
(M W)\end{array}$ & $\begin{array}{c}\Sigma P_{L} \\
(M W)\end{array}$ & $\begin{array}{c}\Sigma P_{G} \\
(M W)\end{array}$ & $(M W)$ \\
\hline \hline $\begin{array}{l}40-42,41-42, \\
34-44,38-65, \\
71-72,24-70\end{array}$ & 228.04 & 1227 & 1076 & 3015 & 3326 \\
\hline \hline
\end{tabular}

It is important to understand that, as the proposed methodology takes into account the BS availability and sufficient generation capability to match the load in each created island, the splitting strategy determined by (14) will include at least one BS unit and will have enough generation capability to match the load in each island. Table IV presents the PPSR constraints included in the methodology. As it can be noticed, there is at least one BS unit and there is more generation capability than load consumption in each island.

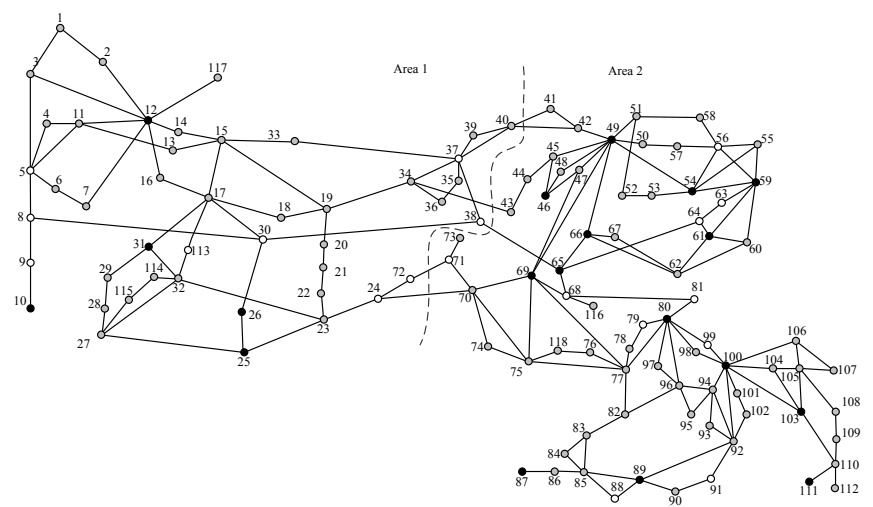

Fig. 6. Splitting strategy into two islands for the IEEE 118-bus test system

TABLE IV

PPSR CONSTRAINTS FOR THE IEEE 118-BUS TEST SYSTEM SPLIT INTO TwO ISLANDS

\begin{tabular}{ccc}
\hline \hline Area number & Available BS units & $\begin{array}{c}\text { Generation capability } \\
(M W)\end{array}$ \\
\hline \hline 1 & 10,25 & 1576 \\
\hline 2 & $69,87,89$ & 4890 \\
\hline
\end{tabular}

2) Splitting the IEEE 118-bus test system into three areas

In order to validate the proposed methodology when the number of areas is greater than two and to demonstrate the recursive bisection approach, the IEEE 118-bus test system is now split into three areas $(r=3)$. As it can be noticed in Fig. 7 , when the power system is split into three islands $(r=3)$, the methodology takes the splitting strategy for two islands $(r=2$ shown in Fig. 6) and determines the new splitting strategy across one of these two previous created areas. In this case, the method splits Area 2 in Fig. 6, across edges 75-77, 76-118, 69-77 and 68-81 to finally obtain Area 3 in Fig. 7.

TABLE V

SPLitTing STRATEGY FOR THE IEEE 118-Bus TeST System SPLit INTO THREE ISLANDS

\begin{tabular}{cc}
\hline \hline Cut-set & $\sum \frac{\left|P_{i j}\right|+\left|P_{j i}\right|}{2}$ \\
& \begin{tabular}{c}
$(M W)$ \\
\hline $40-42,41-43,34-44,38-65,71-72,24-70$
\end{tabular} \\
\hline \hline $75-77,76-118,69-77,68-81$ & 228.04 \\
\hline \hline
\end{tabular}

Table $\mathrm{V}$ presents the objective function (the power flow in transmission lines) for each cut-set when splitting the network into three islands. Table VI finally presents the load and the generation within each island. Blackstart availability and generation capability are also presented in Table VI. As it can be noticed, the generation capability is always larger than the load in each island. Also, BS availability within each island is assured. As these constraints have been included in the proposed method, PPSR is planned in case of a local blackout in any island.

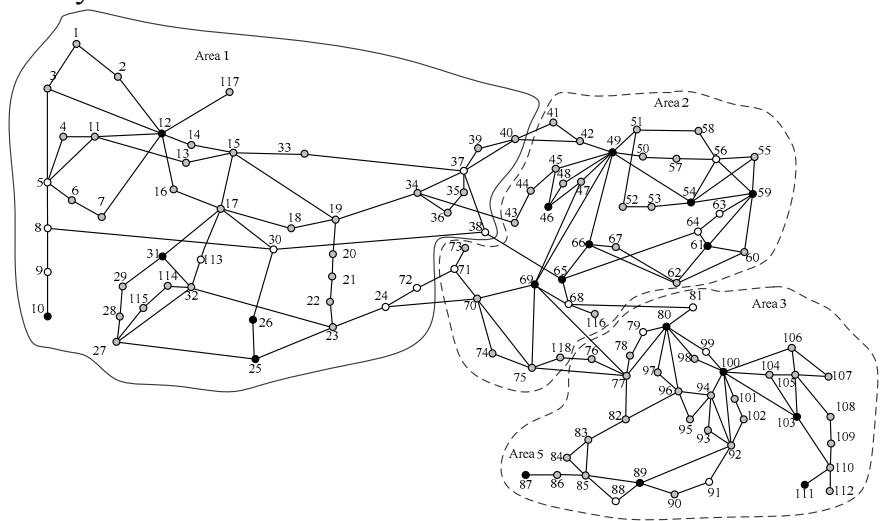

Fig. 7. Splitting strategy into three islands for the IEEE 118-bus test system

TABLE VI

LOAD AND GENERATION WITHIN EACH ISLAND FOR THE IEEE 118-BUS TEST SYSTEM SPLIT INTO THREE ISLANDS

\begin{tabular}{ccccc}
\hline \hline $\begin{array}{c}\text { Area } \\
\text { number }\end{array}$ & $\begin{array}{c}\Sigma P_{L} \\
(M W)\end{array}$ & $\begin{array}{c}\Sigma P_{G} \\
(M W)\end{array}$ & $\begin{array}{c}\text { Available } \\
\text { BS units }\end{array}$ & $\begin{array}{c}\text { Generation } \\
\text { capability } \\
(M W)\end{array}$ \\
\hline \hline 1 & 1227 & 1076 & 10,25 & 1576 \\
\hline 2 & 1651 & 1910 & 69, & 2874 \\
\hline 3 & 1364 & 1416 & 87,89 & 2016 \\
\hline \hline
\end{tabular}

\section{3) Comparing the results with the $O B D D$ algorithm}

For comparison purposes, the algorithm presented in [7] is used. The comparison is carried out for the IEEE 118-bus test system when it is split into two islands. For computational reasons, the OBDD algorithm cannot be applied directly to the IEEE 118-bus test system. Therefore, a reduced network is used to represent this test system when applying the OBDD 
algorithm [7]. The BuDDy package [17] is used in this paper to code the OBDD algorithm.

To find the same splitting strategy, a constraint of $d=250 \mathrm{MW}$ is defined in the objective function (15) of the OBDD. Table VII presents the computation time for the OBDD algorithm and for each step of the proposed method. As it can be noticed the computational time required by the proposed methodology to obtain the solution is considerably shortened.

$$
\left\|\sum_{v_{i} \in \mathbf{V}_{1}}\left(P_{G}^{i}-P_{L}^{i}\right)\right\| \leq d \text { and }\left\|\sum_{v_{j} \in \mathbf{V}_{2}}\left(P_{G}^{j}-P_{L}^{j}\right)\right\| \leq d
$$

TABLE VII

COMPUTATIONAL COMPARISON OF THE PROPOSED METHOD WITH THE OBDD ALGORITHM

\begin{tabular}{ccc}
\hline \hline Step & $\begin{array}{c}\text { Computational time of } \\
\text { the proposed method } \\
(\mathrm{ms})\end{array}$ & $\begin{array}{c}\text { Computational time of } \\
\text { the OBDD algorithm } \\
(\mathrm{ms})\end{array}$ \\
\hline \hline $\begin{array}{c}\text { Weak area } \\
\text { identification }\end{array}$ & 78.1 & - \\
\hline Splitting strategy & $5.0^{*}$ & 9540 \\
\hline Total & $83.1^{*}$ & 9540 \\
\hline \hline
\end{tabular}

*This value includes the power flow computation time

\section{CONCLUSION}

A methodology to split the power system after a large disturbance is proposed in this paper. By determining the weak areas in the power system and splitting the network across the transmission lines with the lowest power exchanged, strong connected islands are created, the power oscillations between areas are reduced, and better loadgeneration is obtained. The weak areas are the links where the methodology is most likely to split the network.

Since the stability margin in the created islands is lower than the one for the entire power system, the created islands might reach the blackout, even after the control actions were intensively carried out. Therefore, in order to restore the operation of a single island, blackstart availability and sufficient generation capability within each created island are included in the proposed methodology. These new constraints can be viewed as a power system restoration planning stage. The method has been successfully tested using two test networks. In these simulations, it has been identified that the loading conditions slightly change the final splitting strategy within the weak areas. This is because the power flow in the transmission lines changes when the operating point changes.

\section{APPENDIX}

The appendix presents generator data (on a 100 MVA base) of the two test systems used in this paper. For the IEEE 9-bus test system, generator data were obtained from [15].

TABLE VIII

GENERATOR DATA FOR THE IEEE 9-BUS TEST SYSTEM

\begin{tabular}{cccccccc}
\hline \hline $\begin{array}{c}\text { Gen. } \\
\text { No. }\end{array}$ & $M_{i}$ & $\begin{array}{c}x_{d, i}^{\prime} \\
(\mathrm{s})\end{array}$ & $\begin{array}{c}P_{\max } \\
(\mathrm{p} . \mathrm{u})\end{array}$ & $\begin{array}{c}\text { Gen. } \\
(M W)\end{array}$ & $\begin{array}{c}M_{i} \\
\text { No. }\end{array}$ & $\begin{array}{c}x_{d, i}^{\prime} \\
(\mathrm{p})\end{array}$ & $\begin{array}{c}P_{\max } \\
(M W)\end{array}$ \\
\hline \hline 1 & 47.28 & 0.0608 & 250 & 3 & 6.02 & 0.1813 & 270 \\
\hline 2 & 12.8 & 0.1198 & 300 & & & & \\
\hline \hline
\end{tabular}

For the IEEE 118-bus test system, generator data were selected according to the typical generator data presented in [11].

TABLE IX

GENERATOR DATA FOR THE IEEE 118-BUS SYSTEM

\begin{tabular}{cccccccc}
\hline \hline $\begin{array}{c}\text { Gen. } \\
\text { No. }\end{array}$ & $\begin{array}{c}M_{i} \\
(\mathrm{~s})\end{array}$ & $\begin{array}{c}x_{d, i}^{\prime} \\
(\mathrm{p} . \mathrm{u})\end{array}$ & $\begin{array}{c}P_{\max } \\
(M W)\end{array}$ & $\begin{array}{c}\text { Gen. } \\
\text { No. }\end{array}$ & $\begin{array}{c}M_{i} \\
(\mathrm{~s})\end{array}$ & $\begin{array}{c}x_{d, i}^{\prime} \\
(\mathrm{p} . \mathrm{u})\end{array}$ & $\begin{array}{c}P_{\max } \\
(M W)\end{array}$ \\
\hline \hline 10 & 23.800 & 0.0592 & 550 & 65 & 30.374 & 0.0668 & 491 \\
\hline 12 & 9.970 & 0.2200 & 185 & 66 & 30.374 & 0.0668 & 492 \\
\hline 25 & 19.210 & 0.1391 & 320 & 69 & 26.944 & 0.0527 & 805 \\
\hline 26 & 19.840 & 0.0961 & 414 & 80 & 26.944 & 0.0527 & 577 \\
\hline 31 & 9.280 & 0.2467 & 107 & 87 & 9.280 & 0.2467 & 104 \\
\hline 46 & 9.280 & 0.2467 & 119 & 89 & 27.360 & 0.0475 & 707 \\
\hline 49 & 19.210 & 0.1391 & 304 & 100 & 22.300 & 0.0948 & 352 \\
\hline 54 & 9.970 & 0.2200 & 148 & 103 & 9.970 & 0.2200 & 140 \\
\hline 59 & 12.680 & 0.1531 & 255 & 111 & 9.970 & 0.2200 & 136 \\
\hline 61 & 12.680 & 0.1531 & 260 & & & & \\
\hline \hline
\end{tabular}

\section{REFERENCES}

M.M. Adibi, P. Clelland, L.H Fink, H. Happ, R.J. Kafka, J. Raine, D. Scheurer, and F. Trefny, "Power system restoration - A task force report," IEEE Transactions on Power Systems, vol. 2, no. 2, pp. 271-277, May 1987.

M.M. Adibi and R.J. Kafka, "Power system restoration issues," IEEE Computer Applications in Power, vol. 4, no. 2, pp. 19-24, Apr. 1991.

U.G. Knight, Power System in Emergencies. London, 2001. H. You, V. Vittal and X. Wang, "Slow Coherency - Based Islanding," IEEE Transactions on Power Systems, vol. 19, no. 1, pp. 483-491, Feb. 2004

S.B. Yusof, G.J. Rogers and R.T.H. Alden, "Slow coherency based network partitioning including load buses," IEEE Transactions on Power Systems, vol. 8, no. 3, pp. 1375-1382, Aug. 1993.

S.S. Lamba and R. Nath, "Coherency identification by the method of weak coupling," Electrical Power \& Energy Systems, vol. 7, no. 4, pp. 233-242, Oct. 1985

K. Sun, D. Zheng and Q. Lu, "Splitting strategies for islanding operation of large-scale power systems using OBDD-based methods," IEEE Transactions on Power Systems, vol. 18, no. 2, pp. 912-922, May 2003.

Q. Zhao, D. Zheng, J. Ma, and Q. Lu, "A study of system splitting strategies for island operation of power system: A two-phase method based on OBDDs," IEEE Transactions on Power Systems, vol. 18, no. 4, pp. 1556-1565, Nov. 2003.

K. Sun, D. Zheng and Q. Lu, "A simulation study of OBDD-based proper splitting strategies for power systems under consideration of transient stability," IEEE Transactions on Power Systems, vol. 20, no. 1, pp. 389-399, Feb. 2005.

V. Terzija, G. Valverde, D. Cai, P. Regulski, V. Madani, L. Fitch, S. Skok, M.M. Begovic, and A.G. Phadke, "Wide-area monitoring, protection, and control of future electric power networks," Proceedings of the IEEE, vol. 99, no. 1, pp. 80-93, Jan. 2011.

P.M. Anderson and A.A. Fouad, Power System Control and Stability, $2^{\text {nd }}$ ed. New York: IEEE Press, 2003.

J.H. Chow, Time-Scale Modeling of Dynamic Networks with Applications to Power Systems vol. 46. New York: SpringerVerlag, 1982.

J. Quiros Tortos, G. Valverde, L. Ding, and V. Terzija, "Optimal Placement of Phasor Measurement Units to Improve Parallel Power System Restoration," presented at the Innovative Smart Grid Technologies, Europe, Manchester, UK, Dec. 2011.

R.A. Date and J.H. Chow, "Aggregation properties of linearized two-time-scale power networks," IEEE Transactions on Circuits and Systems, vol. 38, no. 7, pp. 720-730, Jul. 1991.

P.W. Sauer and M.A. Pai, Power System Dynamic and Stability. New Jersey: Prentice Hall, 1998.

University of Washington, "Power system test case archive," available at: http://www.ee.washington.edu/research/pstca/, consulted in Oct. 2010. 
[17] J. Lind-Nielsen, "BuDDy package," available at: http://sourceforge.net/projects/buddy/, consulted in Jul. 2011.

\section{BIOGRAPHIES}

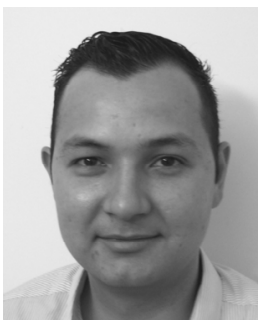

Jairo H. Quirós Tortós (S’08) was born in Limón, Costa Rica. He obtained the B.Sc. and Licentiate degree with honours in Electrical Engineering from the University of Costa Rica, San Pedro, Costa Rica in 2008 and 2009 respectively. Currently he is a $\mathrm{Ph} . \mathrm{D}$. student at The University of Manchester, working on power system restoration and intelligent controlled islanding for power system. His main research interests are application of intelligent methods to power system restoration, and controlled islanding, power system monitoring, protection and control, voltage stability assessment, and power system dynamics. At the end of his Ph.D. studies, he hopes to return to the University of Costa Rica as a Professor and Researcher.

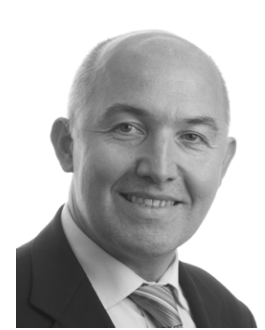

Vladimir Terzija (M'95, SM'2000) is the EPSRC Chair Professor in Power System Engineering in the School of Electrical and Electronic Engineering, The University of Manchester, where he has been since 2006. From 1997 to 1999, he was an Assistant Professor at the University of Belgrade. In 1999, he was awarded a prestigious Humboldt Research Fellowship. From 2000 to 2006, he was with ABB AG, Germany, working as an expert for switchgear and distribution automation. His main research interests are application of intelligent methods to power system monitoring, control, and protection, switchgear and fast transient processes, as well as DSP applications in power systems. 